\title{
A Dual-Channel MAC Protocol for Mobile Ad Hoc Networks
}

\author{
Hongqiang Zhai, Jianfeng Wang, Yuguang Fang and Dapeng Wu \\ Department of Electrical \& Computer Engineering \\ University of Florida, Gainesville, Florida 32611 \\ E-mail: zhai@ecel.ufl.edu,jfwang@ufl.edu,fang@ece.ufl.edu, and wu@ece.ufl.edu
}

\begin{abstract}
Recent studies have shown that the widely used IEEE 802.11 MAC is considerably inefficient in multihop networks. The inefficiency is due to the hidden terminal problem, the exposed terminal problem, the receiver blocking problem, and the intra-flow contention problem. These four problems can lead to "explosion" of control packets and hence poor throughput performance. To address these problems, we propose a novel MAC protocol based upon the IEEE 802.11 MAC. The unique features of the proposed MAC protocol are 1) a system architecture with an out-of-band busy tone and two communication channels, one for control frames and the other for data frames, and 2) a message exchange procedure that provides a comprehensive solution to all the aforementioned four problems. Our studies show that the proposed scheme can simultaneously overcome the four problems, greatly improve spatial reuse, and eliminate the collisions of DATA packets.In addition, the simulation results demonstrate that the new scheme provides a more stable link layer with less control overheads and much less routing failures and significantly improves the throughput, as compared to the IEEE 802.11 MAC.
\end{abstract}

\section{INTRODUCTION}

The IEEE 802.11 MAC [1], a contention based medium access protocol, has been successfully deployed in wireless LANs and has also been implemented in many wireless testbeds and simulation packages for wireless multihop networks. However, the inefficiency of the 802.11 MAC in multihop ad hoc networks has been widely recognized as a serious problem. MAC layer contentions greatly affect the performance of high layer protocols such as TCP and route maintenance [2]-[4].

The source of the above problems comes mainly from the MAC layer. The hidden terminals may introduce collisions and the exposed terminals may lead to low efficiency [6]. In addition to these two notorious problems, the receiver blocking problem, i.e., the intended receiver does not respond the sender with CTS or ACK due to the interference from other ongoing transmissions, also deserves a serious attention. In fact, this problem becomes more severe in the multihop environment and result in inefficiency and starvation of some traffic flows or nodes. Furthermore, for a multihop flow, the nodes along the path have different degree of contention, which results in severe collision and degraded throughput under heavy traffic load. These problems will be detailed in Section II.

Many schemes have been proposed in the current literature to reduce severe collisions of DATA packets at the MAC layer. MACA [5] and MACAW [6] propose the use of RTS and CTS packets for the collision avoidance on the shared channel. MACAW also uses DS packet to advertise the use of the shared channel. However, both of them solve neither the hidden- nor the exposed-terminal problems [13]. The FAMANCS scheme [8] uses long dominating CTS packets to act as a receive busy tone to prevent any competing transmitters in the receiver's range from transmitting. This requires each node hearing the interference to keep quiet for a period of one maximum data packet to guarantee no collision with the ongoing data transmission, which is obviously not efficient especially when the RTS/CTS negotiation process fails or the DATA packet is very short.

Multi-channel random MAC schemes have also been investigated in the past few years [9]-[20]. One common approach to avoid collisions between control packets and data packets is to use separate channels for different kinds of packets. The DCA scheme [10] uses one control channel for RTS/CTS and one or more data channels for DATA/ACK; however, it does not mitigate the hidden terminal problem. The dual busy tone multiple access (DBTMA) schemes ( [14], [15]) use a transmit busy tone to prevent the exposed terminals from becoming new receiver, a receive busy tone to prevent the hidden terminals from becoming new transmitter, and a separate data channel to avoid collisions between control packets and data packets. However, the DBTMA schemes have no acknowledgements for DATA packets which is needed for unreliable wireless links, and the potential collisions between the acknowledgements and other packets can greatly degrade the performance. PAMAS [17] uses a separate control channel to transmit both RTS/CTS packets and busy tone signals. It gives a solution to the hidden terminal problem and mainly focuses on power savings.

Most of the current schemes aggravate the receiver blocking problem when alleviating the hidden terminal problem because the hidden terminals are restricted from transmitting and can not respond its intended sender. The spatial reuse is also degraded since the hidden terminals can not receive as well as not transmit DATA packets. And the flow contention encountered by multihop flows with long paths is not addressed, either, which limits the scalability of MANET.

In this paper, we propose a novel dual-channel MAC protocol with an out-of-band busy tone (DUCHA) for multihop ad hoc networks. Although using a dedicated control channel or busy tone is not a novel idea, DUCHA does not require clock 


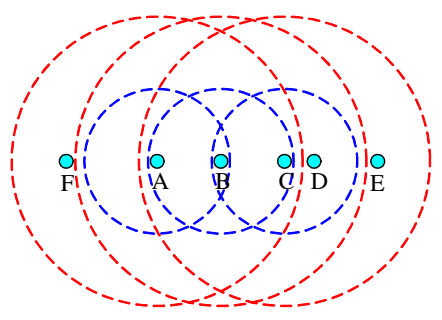

Fig. 1. A simple scenario to illustrate the problems

synchronization among all nodes (as in [20]-[22]) which is especially difficult for a large-scale MANET ( [23], [24]), and it provides a comprehensive solution to all the following four problems: the hidden terminal problem, the exposed terminal problem, the receiver blocking problem, and the intra-flow contention problem (to be defined in Section II-C). There is no collision for DATA packets and the spatial reuse is also greatly improved in DUCHA where not only the exposed terminals can send DATA packets but also the hidden terminals can receive DATA packets.

The rest of this paper is organized as follows. Section II discusses the sources of collisions in the IEEE 802.11 MAC protocol when applied to multihop ad hoc networks, and the ideal protocol behavior we may desire. Section III presents our MAC protocol for multihop ad hoc networks. Simulation results are given in Section IV. Finally, we conclude the paper in Section V.

\section{Problems and The Desired Protocol Behavior}

In this section, we describe a few problems in multi-hop ad hoc networks when the IEEE 802.11 MAC protocol is deployed.

\section{A. Hidden and Exposed Terminal Problems}

The hidden and exposed terminal problems are two well known problems. Here only notice that the transmission range and sensing range should be different in most case. For example, in Fig. 1, the small circles around A, B and C indicate the edges of their individual transmission range and the large circles around them indicate the edges of their individual sensing range. $\mathrm{D}$ is the hidden terminal and $\mathrm{F}$ is the exposed terminal of A when $\mathrm{A}$ is transmitting to $\mathrm{B}$.

\section{B. Receiver Blocking Problem}

The blocked receiver is the one which cannot respond to the RTS intended for this receiver due to other transmission in its sensing range. This may result in unnecessary retransmissions of RTS requests and the subsequent packet discarding, and it may also introduce unfairness. For example as shown in Fig. 1, when $\mathrm{D}$ is transmitting to $\mathrm{E}$, A will not receive the intended CTS from B if it sends RTS to B. This is because B cannot correctly receive A's RTS due to collision from D's transmission. Thus, A keeps retransmitting and doubling contention window until it discards the packet. If $\mathrm{D}$ has a burst of traffic, it will continuously occupy the channel which will starves the flow from A to B.

\section{Intra-Flow Contention Problem}

Intra-flow contention is the contention from the transmissions of packets at upstream and downstream nodes along the path of the same flow. For example, in a single chain topology, the preceding nodes encounter much few contention than the succeeding nodes [25]. Therefore the source, i.e, the first node of the chain, could inject more packets into the chain than the subsequent nodes could forward, which results in packet discarding and severe MAC contentions and make the 802.11 MAC fail to achieve the optimum throughput.

\section{The Desired Protocol Behavior}

The desired MAC protocol for mobile, multihop and wireless ad hoc networks should at least resolve the hidden terminal problem, the exposed terminal problem and the receiver blocking problem. Therefore, the ideal protocol should guarantee that there is only one receiver in the interference range of the transmitter and there is also only one transmitter in the interference range of the receiver. The exposed nodes may start to transmit in spite of the ongoing transmission. The hidden nodes cannot initiate any transmission but may receive packets. Thus, to maximize the spatial reuse or network capacity, it should allow multiple transmitters to transmit in the sensing range of any transmitter and multiple receivers in the sensing range of any receiver to receive. In addition, the transmitter should know whether its intended receiver is blocked or is just outside of its transmission range in case that it does not receive the returned CTS to avoid packet discarding and the wrong protocol behavior at the higher layer, such as unnecessary rerouting requests.

\section{DUCHA: DuAL-Channel MAC Protocol}

In this section, we present the new dual-channel MAC protocol (DUCHA) for multi-hop mobile ad hoc networks. The design is based upon CSMA/CA mechanism in the IEEE 802.11 MAC protocol.

\section{A. Protocol Overview}

To achieve the desired protocol behavior, we utilize dualchannel for control packets and DATA packets, separately. RTS and CTS are transmitted over control channel. Negative CTS (NCTS) is used to solve the receiver blocking problem and is also transmitted in the control channel. DATA is transmitted over the data channel. An out-of-band receiver based busy tone [7] [13] is used to solve the hidden terminal problem. The ACK is not necessary here because our protocol can guarantee that there is no collision for DATA packets. To deal with wireless channel error, we introduce NACK signal which is continuing busy tone signal when the receiver determines that the received DATA packet is corrupted. The sender will not misinterpret this NACK signal because there are no other receivers in its sensing range and hence no interfering NACK signals and will conclude that the transmission is successful 


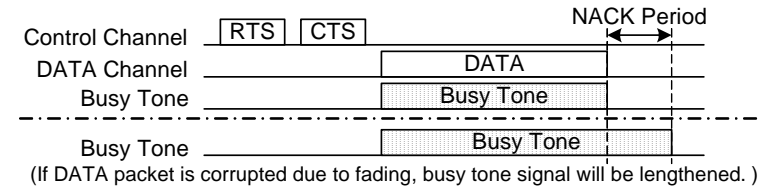

Fig. 2. Proposed protocol

if no NACK signal is sensed. The basic message exchange sequence is shown in Fig. 2.

\section{B. Basic Message Exchange}

1) RTS: Any node must senses the control channel idle at least for DIFS long and senses no busy tone signal before initiating new transmission of an RTS. If it senses the noisy (busy) control channel longer than or equal to the RTS period, it should defer long enough (at least for SIFS + CTS $+2 \times$ max-propagation-delay) to avoid possible collision with the CTS's reception at some sender. For example, in Fig. 1, when A finishes transmitting its RTS to B, F should wait before transmitting at least long enough for A to finish receiving the possible returning CTS/NCTS from B.

2) CTS/NCTS : Any node which correctly receives the RTS should return CTS after SIFS spacing regardless the control channel status if the DATA channel is idle.

If both control and DATA channel are busy, it ignores the RTS to avoid possible interference to the CTS's reception at other RTS's transmitter. Since the data channel is not available, there should not have any collision with CTS/NCTS to other possible competitor using the data channel.

If control channel is idle for at least one CTS packet long and the DATA channel is busy, it returns NCTS. The NCTS estimates the remaining DATA transmission time in its duration field according to the difference between the transmission time of maximum DATA packet and the length it has sensed the DATA channel.

3) DATA: RTS's transmitter should start DATA transmission after correctly receiving the CTS if no busy tone signal is sensed. If the sender receives a NCTS, it defers its transmission according to the duration field of NCTS. Otherwise, it assumes there is a collision occurred, will then double its backoff window and defer its transmission.

4) Busy Tone: The intended receiver begins to sense the data channel after it transmits CTS. If the receiver does not receive signal with enough power in the data channel in the due time that the first few bits of the DATA packet reaches it, it will assume the sender does not transmit DATA and finish the receiving procedure. Otherwise, it transmits busy tone signal to prevent possible transmission from hidden terminals.

5) NACK : The intended receiver has a timer to indicate when it should finish the reception of the DATA packet according to the duration field in the previously received RTS. If the timer expires and has not received the correct DATA packet, it assumes the DATA transmission fails and sends NACK by continuing the busy tone signal for an appropriate period. If it correctly receives the DATA packet, it stops the busy tone signal and finishes the receiving procedure.

The sender assumes its DATA transmission is successful if there is no NACK signal sensed during the NACK period. Otherwise, it assumes its transmission fails because of wireless channel error and then starts the retransmission procedure.

In the following few subsections, we use examples to illustrate how our DUCHA solves those well-know problems.

\section{Solution to the hidden terminal problem}

As shown in Fig. 1, B broadcasts busy tone signal when it receives DATA packet from A. The hidden terminal of A, i.e., $\mathrm{D}$, could hear B's busy tone signal and thus will not transmit anything in the DATA channel to interfere with B's reception. Thus the busy tone signal from the DATA's receiver prevents any hidden terminals of the intended sender to interfere with the reception. Moreover, no DATA packets are dropped due to the hidden terminal problem.

\section{Solution to the exposed terminal problem}

In Fig. $1, \mathrm{~B}$ is the exposed terminal of $\mathrm{D}$ when $\mathrm{D}$ is transmitting DATA packet to E. B could initiate RTS/CTS exchange with A though it can sense D's transmission in the DATA channel. After the RTS/CTS exchange is successful between B and A, B begins to transmit DATA packet to A. Since $A$ is out of the sensing range of $D$ and $E$ is out of sensing range of $\mathrm{B}$, both $\mathrm{A}$ and $\mathrm{E}$ could correctly receive the DATA packet destined to them. Thus, the exposed terminal problem could transmit DATA packets which could greatly enhance the spatial reuse ratio.

\section{E. Solution to the receiver blocking problem}

In Fig. 1, B is the blocked receiver in the IEEE 802.11 MAC protocol when D is transmitting DATA packets to E. In our protocol DUCHA, B can correctly receive A's RTS in the control channel while D sends DATA packets in the DATA channel. Then B returns NCTS to A because it senses busy medium in the DATA channel. The duration field of NCTS estimates the remaining busy period in the DATA channel which takes to finish D's transmission. When A receives the NCTS, it defers its transmission and stop the unnecessary retransmissions. It retries the transmission after the period indicated in the duration field of NCTS. Once the RTS/CTS exchange is successful between A and B, A begins to transmit DATA packet to B. B will correctly receive the DATA packet because there is no hidden terminal problem for receiving DATA packets.

\section{F. Maximum spatial reuse}

As discussed above, the exposed terminals could transmit DATA packets. Furthermore, in our protocol, the hidden terminal could receive DATA packets though it cannot transmit. In Fig. 1, D is the hidden terminal of A when A is transmitting DATA packet to B. After the RTS/CTS exchange between E and D is successful in the control channel, E could transmit DATA packets to D. Since D is out of A's sensing range and B 
is out of E's sensing range, both D and E could correctly receive the intended DATA packets. Thus our protocol DUCHA could achieve maximum spatial reuse by allowing multiple transmitters or multiple receivers in the sensing range of each other. At the same time, there are no collisions for DATA packets as well as the NACK signals because there is only one transmitter in its intended receiver's sensing range and only one receiver in its intended transmitter's sensing range.

\section{G. Inherent Mechanisms to solve the intra-flow contention problem}

In our DUCHA protocol, the receiver of DATA packets have the highest priority to access the channel for next DATA transmission. When one node correctly receives a DATA packet, it could immediately start the backoff procedure for the new transmission while the upstream and downstream nodes in its sensing range are prevented from transmitting DATA packets during the NACK period. In fact, this could achieve optimum packet scheduling for chain topology and it is similar for any single flow scenario.

For example, in a chain topology consisting of node 0,1 , $2, \ldots, 8$ from left to right, node 1 has the highest priority to access the channel when it receives one packet from node 0 and hence immediately forwards the packet to node 2 . For the same reason, node 2 immediately forwards the received packet to node 3. Then node 3 forwards the received packet to node 4. Because node 0 can sense node 1 and 2's transmissions, it will not interfere with these two nodes. Node 0 could not send packets to node 1 either when node 3 forwards packet to 4 because node 1 is in the interference range of node 3 . When node 4 forwards packet to 5 , node 0 could have chance to send packet to node 1 . In general, nodes which are 4 hops away from each other along the path could simultaneously send packets to their next hops. Thus the procedure could utilize $1 / 4$ of the channel bandwidth, the maximum throughput which can be approached by the chain topology [25]

\section{H. Notes on the proposed protocol}

There is no collisions for DATA packets in the proposed protocol because there is only one DATA transmitter in the sensing range of any ongoing receiver in the DATA channel. The out-of-band busy tone signal prevents any hidden nodes from initiating new DATA transmission in the DATA channel.

There is no collision for NACK signal, i.e., the continuing busy tone, either, because there is only one DATA receiver in the sensing range of any ongoing sender in the DATA channel. After successful RTS/CTS exchange between the sender and its intended receiver, all nodes in the sensing range of the sender can sense its transmission in the DATA channel and thus are restricted from becoming DATA transmitters.

The control overhead could be reduced although we introduce a new NCTS packet and a new NACK signal. First, NCTS is only transmitted when the intended receiver can not receive DATA packet. It can save a lot of unnecessary retransmitted RTS packets as discussed in Section III-E. Second, NACK signal occurs only when the DATA packet is corrupted

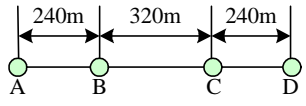

Fig. 3. One simple topology

due to channel fading, and hence its transmission frequency is also much smaller than that of ACK packets in the 802.11 MAC protocol. Third, there is no collision for DATA packets and hence the transmissions of RTS and CTS for corrupted DATA packets are saved.

\section{Performance Evaluation}

\section{A. Simulation Environments}

We now evaluate the performance of our protocol and compare it with the IEEE 802.11 scheme. The simulation tool is one of the widely used network simulation tools $n s 2$. We use pre-computed shortest path with no routing overhead. The propagation model is the two-ray ground model. The transmission range of each node is approximately $250 \mathrm{~m}$ and the sensing/interference range is approximately $550 \mathrm{~m}$ according to the default value of the received power threshold and the carrier sensing threshold. For comparison purpose, the simulations use the same total bandwidth, i.e., $2 \mathrm{Mbps}$, for both 802.11 and DUCHA. Other default values of important parameters are shown in Table I. In the following figures, our protocol will be referred to as Dual-channel MAC protocol (DUCHA).

TABLE I

Default Values in the Simulations

\begin{tabular}{|l|l|}
\hline Preamble of all kinds of packets & $192 \mu \mathrm{s}$ \\
\hline Control channel speed in DUCHA & $0.3 \mathrm{Mbps}$ \\
\hline Data channel speed in DUCHA & $1.7 \mathrm{Mbps}$ \\
\hline DATA rate in 802.11 & $2.0 \mathrm{Mbps}$ \\
\hline Length of NACK signal & $150 \mu \mathrm{s}$ \\
\hline DATA Packet size & 1000 Bytes \\
\hline
\end{tabular}

\section{B. Simple Scenarios}

To verify the correctness of our protocol, we first investigate one simple scenario shown in Fig. 3, where there are hidden terminals, exposed terminals and receiver blocking problems if IEEE 802.11 MAC protocol is used.

1) Hidden terminals: There are two flows with the same CBR traffic: flow 1 is from A to B and flow 2 is from C to D. C is a hidden terminal of A and cannot sense A's transmission or cannot correctly receive B's CTS. However, C's transmission will introduce enough interference at node $\mathrm{B}$, which would affect B's reception.

Fig. 4(a) shows that the number of collided DATA packets increases with the offered load in IEEE 802.11 while our protocol has no collision for the DATA packets. This in fact verifies that there is no hidden terminal problem for the transmission of DATA packets in our protocol. The reason is that B's busy tone signal prevents the hidden terminal C 


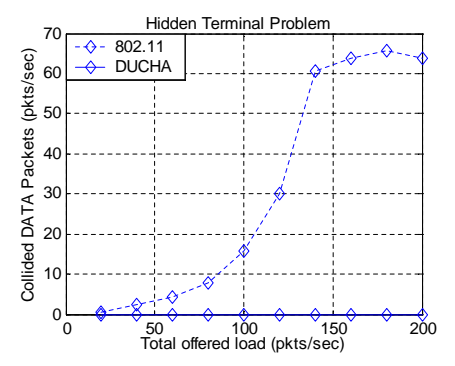

(a)

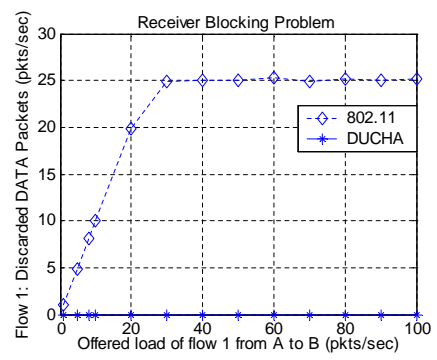

(c)

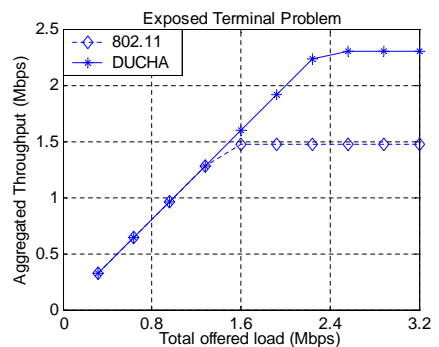

(b)

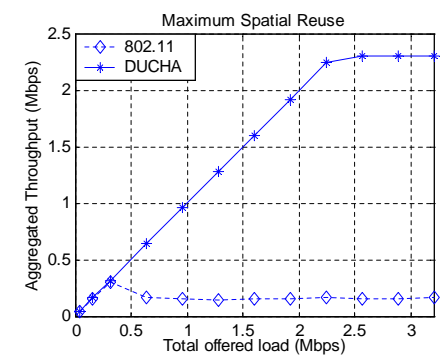

(d)
Fig. 4. Simulation results for the simple topology

from transmitting and hence there is no collision at $\mathrm{B}$ and hence $B$ can still receive A's DATA packets. However, in the IEEE 802.11 MAC protocol, $\mathrm{C}$ has no way to know that $\mathrm{A}$ is transmitting DATA packets to $\mathrm{B}$ and hence cause collisions at $\mathrm{B}$ if $\mathrm{C}$ begins transmissions.

2) Exposed terminals: We now examine the exposed terminal problem. Assume that there are two flows with the same CBR traffic: one is from $\mathrm{B}$ to $\mathrm{A}$ and another is from $\mathrm{C}$ to $\mathrm{D}$. $\mathrm{B}$ and $\mathrm{C}$ are the exposed terminals of each other. For example, B can sense C's transmission but not D's transmission and B will not interfere with D's reception.

In IEEE 802.11 MAC protocol, B and C cannot transmit DATA packets at the same time while they can in our DUCHA protocol. So our protocol should have much higher aggregated throughput in this simple scenario under heavy offered load. The improvement is about $55 \%$ as shown in Fig. 4(b).

3) Receiver blocking problem: The topology remains the same except $\mathrm{C}$ always has packets to transmit to $\mathrm{D}$. When $\mathrm{C}$ is transmitting to $\mathrm{D}, \mathrm{B}$ is the blocked receiver. It cannot respond to A's RTS, which will lead to packet discarding.

Fig 4(c) shows that in IEEE 802.11 the sender A, whose intended receiver $\mathrm{B}$ is blocked, cannot successfully transmit any packets. This is because that B could not correctly receive A's RTS and thus A continuously discards DATA packets after multiple transmission failures of RTS packets. While in our protocol DUCHA, the control packets are transmitted in a separate channel and the blocked receiver could return an NCTS packet to its intended sender during the period of neighboring DATA transmissions. Furthermore, in our protocol, A can obtain a part of the bandwidth to transmit DATA packets while in IEEE 802.11, A's DATA transmissions will be corrupted by its hidden terminal $C$ even if the RTS-CTS exchange is successful between A and B.

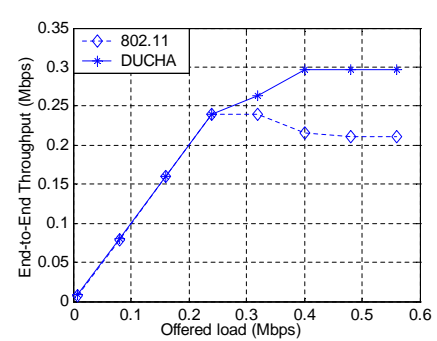

(a) Chain topology

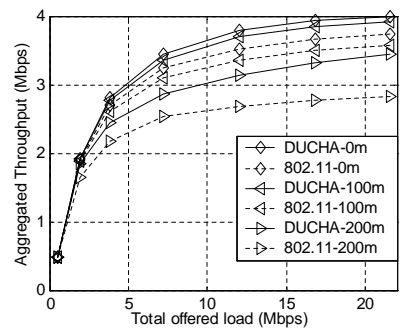

(b) Random topology
Fig. 5. 9-node chain topology and one-hop flows in random topology

4) Maximum spatial reuse: Our DUCHA protocol could allow the hidden terminal to receive DATA packets as well as to allow the exposed terminal to transmit DATA packets to improve the spatial reuse. In the simulation, there are two flows with the same CBR traffic: flow 1 is from $A$ to $B$ and flow 2 is from $\mathrm{D}$ to $\mathrm{C}$.

Fig 4(d) shows that our protocol has much higher aggregated throughput than IEEE 802.11 MAC. The latter suffers not only from the poor spatial reuse but also from the collisions among RTS, CTS, DATA and ACK packets since B and C are hidden terminals of A and D, respectively.

5) Intra-flow contention: Our protocol DUCHA could mitigate the intra-flow contention as discussed in section III-G. Fig. 5(a) shows the aggregated throughput of a 9-node chain topology. DUCHA improves the maximum throughput by about $25 \%$ and has a $40 \%$ higher throughput than IEEE 802.11 MAC under heavy offered load. This is because DUCHA has a large spatial reuse ratio in the DATA channel and could achieve the optimum packet scheduling for the chain topology independent of the traffic load while IEEE 802.11 MAC suffers from collisions under heavy load.

\section{Random Topology for One-hop Flows}

In this simulation study, 60 nodes are randomly placed in a $1000 \mathrm{~m} \times 300 \mathrm{~m}$ area. Each node has the same CBR traffic and randomly selects one neighbor as the destination, which is at least the minimum source-destination distance, i.e., 0, 100, $200 \mathrm{~m}$, far apart. All results are averaged over 30 random simulations.

We observe from Fig. 5(b) that the aggregated throughput for all flows decreases when the minimum source-destination distance increases. The aggregated throughput of our protocol is higher than that of IEEE 802.11 MAC. And it degrades much slower in our protocol than in IEEE 802.11 MAC and it is improved by about $8 \%$ to $28 \%$ when the minimum sourcedestination distance increases from $0 \mathrm{~m}$ to $200 \mathrm{~m}$.

This is reasonable. For example, A and B are the sourcedestination pair. The larger the distance between $\mathrm{A}$ and $\mathrm{B}$, the larger the hidden area where nodes cannot sense A's transmission but can sense B's transmission. So in IEEE 802.11 MAC, the hidden terminal problem becomes more severe when the distance between $\mathrm{A}$ and $\mathrm{B}$ becomes larger. On the other hand, in IEEE 802.11 MAC, all the nodes in the sensing range 
of A or B should not transmit anything, i.e., both sensing ranges of $\mathrm{A}$ and $\mathrm{B}$ could not be reused by other transmissions. However, in our protocol DUCHA, the exposed area where nodes can sense the sender's transmission but not the receiver's transmission could be reused for new senders, and the hidden area where nodes can sense the receiver's transmission but not the sender's transmission could be reused for new receivers. Thus the larger the source-destination distance is, the higher the system capacity our protocol DUCHA could obtain than the IEEE 802.11 MAC.

In fact, most of the current routing algorithms maximize the distance between the upstream node and the downstream node when selecting a path to reduce the hop-count, the delay and the power consumption for delivering the packets from the source to the destination. Our protocol DUCHA also gives a good solution to the intra-flow contention problem and could achieve optimum packet scheduling for the chain topology. Thus our protocol will be more friendly to multihop flows.

\section{COnClusions}

In this paper, we identified four problems that cause dramatic performance degradation of the IEEE 802.11 MAC in multihop ad hoc networks, namely, the hidden terminal problem, the exposed terminal problem, the receiver blocking problem, and the intra-flow contention problem. To mitigate these problems, we proposed a new MAC protocol called DUCHA. Our DUCHA protocol uses two channels: one for control packets and the other for DATA packets; a busy tone signal is used to solve the hidden terminal problem and also used to transmit the NACK signal if necessary. Our DUCHA protocol also uses NCTS to notify a sender that its intended receiver is blocked and cannot receive DATA packets; in contrast, the IEEE 802.11 MAC cannot distinguish the receiver blocking from destination being unreachable. Moreover, DUCHA is more friendly to the routing layer since DUCHA can provide accurate next-hop information, leading to reduction in the number of unnecessary rerouting requests. Besides, DUCHA has the following nice features: 1) no collisions for DATA packets and NACK signal, 2) much less control packets and discarded DATA packets, and 3) achieving much higher spatial reuse. More importantly, DUCHA can simultaneously overcome the four problems that cause inefficiency of the IEEE 802.11 MAC.

Extensive simulations indicate that DUCHA eliminates the collisions of DATA packets, greatly increases the spatial reuse, and hence significantly improves the throughput as compared to the IEEE 802.11 MAC. In addition, by solving the intraflow contention problem, DUCHA is more scalable for large networks and maintain high resource utilization ratio for multihop flows while the performance of the IEEE 802.11 MAC degrades significantly when the flows have long paths.

\section{ACKNOWLEDGMENT}

This work was supported in part by the U.S. Office of Naval Research under Young Investigator Award N000140210464 and under grant N000140210554.

\section{REFERENCES}

[1] IEEE standard for Wireless LAN Medium Access Control (MAC) and Physical Layer (PHY) specifications, ISO/IEC 8802-11: 1999(E), Aug. 1999

[2] J. Broch, D.A. Maltz, D.B. Johnson, Y. Hu, and J. Jetcheva, "A performance Comparison of Multihop Wireless Ad Hoc Network Routing Protocols," Proc. ACM MobiCom, Oct. 1998.

[3] C. Perkins, E.M. Royer, S.R. Das, and M.K. Marina, "Performance Comparison of Two On-demand Routing Protocols for Ad Hoc Networks," IEEE Personal Communications, pp. 16-28, Feb. 2001.

[4] S. Xu and T. Safadawi, "Does the IEEE 802.11 MAC Protocol Work Well in Multihop Wireless Ad Hoc Networks?" IEEE Communications Magazine, pp. 130-137, June 2001.

[5] P. Karn, "MACA-A new channel access method for packet radio," ARRL/CRRL Amateur Radio 9th Computer Networking Conf., 1990.

[6] V. Bharghavan, A. Demers, S. Shenker, and L. Zhang, "MACAW: A media access protocol for wireless LAN's," Proc. ACM SIGCOMM, 1994.

[7] F.A. Tobagi and L. Kleinrock, "Packet switching in radio channels: Part II-The hidden terminal problem in carrier sense multiple-access and the busy-tone solution," IEEE Trans. Commun., vol. COM-23, pp. 14171433, Dec. 1975

[8] C. L. Fullmer and J.J. Garcia-Luna-Aceves, "Solutions to Hidden Terminal Problems in Wireless Networks," Proc. ACM SIGCOMM'97, September, 1997.

[9] F. Tobagi, L. Kleinrock, "Packet Switching in Radio Channels: Part III C Polling and (Dynamic) Split-Channel Reservation Multiple Access", IEEE Trans. Commun, Vol. COM-24, no. 8, pp. 832-844, August 1976.

[10] S.-L. Wu, C.-Y. Lin, Y.-C. Tseng, and J.-P. Sheu, "A New Multi-Channel MAC Protocol with On-Demand Channel Assignment for Mobile Ad Hoc Networks," Int'l Symp. on Parallel Architectures, Algorithms and Networks (I-SPAN), pp. 232-237, 2000.

[11] N. Choi, Y. Seok, and Y. Choi, "Multi-channel MAC Protocol for Mobile Ad Hoc Networks," Proc. IEEE VTC 2003-Fall, October, 2003.

[12] S.-R. Ye, Y.-C. Wang, and Y.-C. Tseng, "A jamming-based MAC protocol for wireless multihop ad hoc networks," in Proc. VTC 2003Fall, October, 2003

[13] Z.J. Haas and J. Deng, "Dual Busy Tone Multiple Access (DBTMA) - A Multiple Access Control for Ad Hoc Networks," IEEE Trans. Commun, vol. 50, no. 6, pp. 975-985, June 2002.

[14] Z.J. Haas and J. Deng, "Dual Busy Tone Multiple Access (DBTMA): Performance Results,'”in Proc. IEEE WCNC'99, September 1999.

[15] S. Wu, Y. Tseng and J. Sheu, "Intelligent medium access for mobile ad hoc networks with busy tones and power control," IEEE Journal on Selected Areas in Communications, Vol. 18, pp. 1647 -1657, Sept. 2000.

[16] K. Liu, T. Wong, J. Li, L. Bu, and J. Han, "A reservation-based multiple access protocol with collision avoidance for wireless multihop ad hoc networks," Proc. IEEE ICC'03, May, 2003.

[17] S. Singh and C. S. Raghavendra, "PAMAS - Power Aware MultiAccess Protocol with Signalling for Ad Hoc Networks," ACM Computer Communications Review, July 1998.

[18] A. Muqattash and M. Krunz, "Power Controlled Dual Channel (PCDC) Medium Access Protocol for Wireless Ad Hoc Networks," Proc. IEEE INFOCOM'03, March 2003.

[19] Y. Li, H. Wu, D. Perkins, N. Tzeng, and M. Bayoumi, "MAC-SCC: Medium Access Control with a Separate Control Channel for Multihop Wireless Networks," 23rd International Conference on Distributed Computing Systems Workshops (ICDCSW'03), May 2003.

[20] T. You, C. Yeh, and H. Hassanein, "A New Class of Collision Prevention MAC Protocols for Wireless Ad Hoc Networks,' Proc. IEEE ICC'03, May 2003.

[21] L. Bao and J.J. Garcia-Luna-Aceves. Hybrid Channel Access Scheduling in Ad Hoc Networks. Proc. IEEE ICNP'02, Nov. 2002.

[22] J. So and N. H. Vaidya, "A Multi-Channel MAC Protocol for Ad Hoc Wireless Networks," Proc. ACM Mobihoc'04, May, 2004.

[23] K. Romer, "Time Synchronization in Ad Hoc Networks," Proc. ACM MobiHoc'1, Oct. 2001

[24] S. Ganeriwal, R. Kumar, M.B. Srivastava, "Timing-sync Protocol for Sensor Networks," Proc. ACM Sensys'03, Nov. 2003

[25] J. Li, C. Blake, D. S. J. De Couto, H. I. Lee and R. Morris, "Capacity of Ad Hoc Wireless Network," Proc. ACM MobiCom'01, July 2001. 